\title{
Detection of hydrogen fluoride absorption in diffuse molecular clouds with Herschel/HIFI: an ubiquitous tracer of molecular gas ${ }^{\star}$
}

\author{
P. Sonnentrucker ${ }^{1}$, D. A. Neufeld ${ }^{1}$, T. G. Phillips ${ }^{2}$, M. Gerin ${ }^{3}$, D. C. Lis ${ }^{2}$, M. De Luca ${ }^{3}$, J. R. Goicoechea ${ }^{4}$, \\ J. H. Black ${ }^{5}$, T.A. Bell ${ }^{2}$, F. Boulanger ${ }^{6}$, J. Cernicharo ${ }^{4}$, A. Coutens ${ }^{7}$, E. Dartois ${ }^{6}$, M. Kaźmierczak ${ }^{8}$, P. Encrenaz ${ }^{3}$, \\ E. Falgarone ${ }^{3}$, T. R. Geballe ${ }^{9}$, T. Giesen ${ }^{10}$, B. Godard ${ }^{3}$, P. F. Goldsmith ${ }^{11}$, C. Gry ${ }^{12}$, H. Gupta ${ }^{11}$, P. Hennebelle ${ }^{3}$, \\ E. Herbst ${ }^{13}$, P. Hily-Blant ${ }^{14}$, C. Joblin ${ }^{7}$, R. Kołos ${ }^{15}$, J. Krełowski ${ }^{8}$, J. Martín-Pintado ${ }^{4}$, K. M. Menten ${ }^{16}$, R. Monje ${ }^{2}$, \\ B. Mookerjea ${ }^{17}$, J. Pearson ${ }^{11}$, M. Perault ${ }^{3}$, C. M. Persson ${ }^{5}$, R. Plume ${ }^{18}$, M. Salez ${ }^{3}$, S. Schlemmer ${ }^{10}$, M. Schmidt ${ }^{19}$, \\ J. Stutzki ${ }^{10}$, D. Teyssier ${ }^{20}$, C. Vastel ${ }^{7}$, S. Yu ${ }^{11}$, E. Caux ${ }^{7}$, R. Güsten ${ }^{16}$, W. A. Hatch ${ }^{11}$, T. Klein ${ }^{16}$, I. Mehdi ${ }^{11}$, \\ P. Morris ${ }^{21}$, and J. S. Ward ${ }^{11}$
}

(Affiliations are available on page 5 of the online edition)

Received 29 May 2010 / Accepted 13 July 2010

\begin{abstract}
We discuss the detection of absorption by interstellar hydrogen fluoride (HF) along the sight line to the submillimeter continuum sources W49N and W51. We have used Herschel's HIFI instrument in dual beam switch mode to observe the $1232.4762 \mathrm{GHz} J=1-0 \mathrm{HF}$ transition in the upper sideband of the band 5a receiver. We detected foreground absorption by HF toward both sources over a wide range of velocities. Optically thin absorption components were detected on both sight lines, allowing us to measure - as opposed to obtain a lower limit on - the column density of $\mathrm{HF}$ for the first time. As in previous observations of HF toward the source G10.6-0.4, the derived HF column density is typically comparable to that of water vapor, even though the elemental abundance of oxygen is greater than that of fluorine by four orders of magnitude. We used the rather uncertain $N(\mathrm{CH})-N\left(\mathrm{H}_{2}\right)$ relationship derived previously toward diffuse molecular clouds to infer the molecular hydrogen column density in the clouds exhibiting HF absorption. Within the uncertainties, we find that the abundance of $\mathrm{HF}$ with respect to $\mathrm{H}_{2}$ is consistent with the theoretical prediction that $\mathrm{HF}$ is the main reservoir of gas-phase fluorine for these clouds. Thus, hydrogen fluoride has the potential to become an excellent tracer of molecular hydrogen, and provides a sensitive probe of clouds of small $\mathrm{H}_{2}$ column density. Indeed, the observations of hydrogen fluoride reported here reveal the presence of a low column density diffuse molecular cloud along the W51 sight line, at an LSR velocity of $\sim 24 \mathrm{~km} \mathrm{~s}^{-1}$, that had not been identified in molecular absorption line studies prior to the launch of Herschel.
\end{abstract}

Key words. molecular processes - astrochemistry - ISM: molecules - submillimeter: ISM

\section{Introduction}

In some of the first results obtained using the HIFI instrument (de Graauw et al. 2010) on Herschel (Pilbratt et al. 2010), the detection of hydrogen fluoride has been reported along sight lines to the bright continuum source G10.6-0.4 (Neufeld et al. 2010) and the Orion hot core (Phillips et al. 2010). In addition, absorption by HF has been detected (although spectrally unresolved) in the spectrum of G29.96-0.02, even at the lower spectral resolution of the SPIRE instrument (Kirk et al. 2010). For G10.6-0.4, observations of the $J=1-0$ transition of HF revealed optically thick absorption in foreground gas clouds unassociated with the continuum source, and implied a lower limit of $30 \%$ for the fraction of gas-phase fluorine nuclei in HF. Remarkably, the inferred hydrogen fluoride abundance was comparable to that of water vapor, even though the interstellar fluorine abundance is four orders of magnitude lower than that of oxygen. These observations corroborated a theoretical prediction (Neufeld et al. 2005;

* Herschel is an ESA space observatory with science instruments provided by European-led Principal Investigator consortia and with important participation from NASA.
Neufeld \& Wolfire 2009) that HF is the dominant reservoir of fluorine wherever the interstellar $\mathrm{H}_{2}$ /atomic $\mathrm{H}$ ratio exceeds $\sim 1$; the unusual behavior of fluorine is explained by its unique thermochemistry, $\mathrm{F}$ being the only atom in the periodic table that can react exothermically with $\mathrm{H}_{2}$ to form a hydride. An implication of this theoretical prediction, together with the detection of strong HF absorption along the sight lines to G10.6-0.4, the Orion hot core, and G29.96-0.02, is that HF may serve as a valuable surrogate tracer for molecular hydrogen within the diffuse interstellar medium, both in the Milky Way and other galaxies.

As part of the PRISMAS (PRobing InterStellar Molecules with Absorption line Studies) key program, we have observed hydrogen fluoride toward two additional Galactic continuum sources with sight lines that intersect foreground gas clouds: the star-forming regions $\mathrm{W} 49 \mathrm{~N}$ and W51, located at distances of $\sim 11.4$ (Gwinn et al. 1992) and $2.4 \mathrm{kpc}$ (Sato et al. 2010) from the Sun, respectively. Previous observations of both sources have led to the detection of foreground absorption by many species, including atomic hydrogen (e.g. Fish et al. 2003), $\mathrm{OH}, \mathrm{H}_{2} \mathrm{O}$ (Neufeld et al. 2002; Plume et al. 2004), $\mathrm{HCO}^{+}, \mathrm{CN}, \mathrm{HCN}$, and HNC (Godard et al. 2010). In general, many of the molecular 
absorption features observed previously toward W49N, and particularly toward W51, are less saturated than those measured toward G10.6-0.4; this difference makes W49N and W51 attractive targets for observations of $\mathrm{HF}$ absorption, because our previous study of G10.6-0.4 yielded only a lower limit on the HF abundance, as the observed absorption line was completely saturated over most velocities. In this letter we present the results of observations of HF $J=1-0$ obtained toward W49N and W51.

\section{Observations and data analysis}

The $J=1-0$ transition of $\mathrm{HF}$, with rest frequency 1232.4762 GHz (Nolt et al. 1987), was observed toward W49N and W51 on 2010 March 22 in the upper sideband of HIFI receiver band $5 \mathrm{a}$. The observations were carried out at three different local oscillator (LO) tunings in order to securely identify the HF line toward both sight lines. The dual beam switch mode (DBS) was used with a reference position located $3^{\prime}$ on either side of the source position along an East-West axis. We centered the telescope beam at $\alpha=19 \mathrm{~h} 10 \mathrm{~m} 13.2 \mathrm{~s}, \delta=09^{\circ} 06^{\prime} 12.0^{\prime \prime}$ for $\mathrm{W} 49 \mathrm{~N}$ and $\alpha=19 \mathrm{~h} 23 \mathrm{~m} 43.9 \mathrm{~s}, \delta=14^{\circ} 30^{\prime} 30.5^{\prime \prime}$ for W51 (J2000.0). The total on-source integration time amounts to $222 \mathrm{~s}$ on each source using the wide band spectrometer (WBS) that offers a spectral resolution of $1.1 \mathrm{MHz}\left(\sim 0.3 \mathrm{~km} \mathrm{~s}^{-1}\right.$ at $\left.1232 \mathrm{GHz}\right)$.

The data were processed to Level 2 using the standard HIFI pipeline and HIPE version 2.4 (Ott 2010), hence providing fully calibrated spectra for both polarization modes at each LO setting. We further analyzed the Level 2 data using IDL routines developed locally. We found that the signals measured in the two polarization modes for each LO setting were in excellent agreement, as were the spectra obtained at the three LO tunings. We therefore produced an average spectrum that consists of the weighted sum of all six observations (three LO tunings with two polarization modes each), where each observation is weighted in proportion to its signal-to-noise ratio. The resulting double sideband continuum antenna temperatures are $T_{\mathrm{A}}$ (cont) $=10.65 \mathrm{~K}$ for $\mathrm{W} 49 \mathrm{~N}$ and $T_{\mathrm{A}}$ (cont) $=10.17 \mathrm{~K}$ for $\mathrm{W} 51$. The rms noise was $0.13 \mathrm{~K}$ for both sources.

HIFI employs double sideband receivers. Thus for a sideband gain ratio of unity, the complete absorption of radiation at a single frequency will reduce the measured antenna temperature to one-half the apparent continuum level. Assuming the sideband gain ratio is equal to unity, the flux normalized with respect to the continuum flux can be expressed as $\left[T_{\mathrm{A}}-\right.$ $0.5 T_{\mathrm{A}}$ (cont) $] / 0.5 T_{\mathrm{A}}$ (cont). The upper panels of Figs. 1 and 2 show the normalized fluxes versus Doppler velocity in the LSR frame $\left(V_{\mathrm{LSR}}\right)$ for $\mathrm{HF}$ (black line) and para-water (green line) toward $\mathrm{W} 49 \mathrm{~N}$ and $\mathrm{W} 51$, respectively. The para- $\mathrm{H}_{2} \mathrm{O} 1_{11}-0_{00}$ $(v=1113.343 \mathrm{GHz})$ spectra presented here for comparison were observed in the lower sideband of receiver $5 \mathrm{a}$ in the same observing campaign as HF. The total on-source integration time was $87.6 \mathrm{~s}$ for each target. The methods we used to reduce the $\mathrm{HF}$ data were also applied to the para-water data (see above). The dashed lines represent the continuum temperature $T_{\mathrm{A}}$ (cont) (K) normalized to unity and the zero flux level. One can see that toward both sight lines the sideband gain ratios are indeed consistent with unity.

\section{Results}

The spectra shown in Figs. 1 and 2 reveal the presence of HF (black line) and para-water (green line) absorption in a number of foreground clouds along the sight lines to both W49N and
W51. The presence of molecular absorption in these spectra was anticipated by previous observations (e.g., Neufeld et al. 2002; Plume et al. 2004; Godard et al. 2010).

For W49N, the foreground absorptions in the velocity range -10 to $\sim 25 \mathrm{~km} \mathrm{~s}^{-1}$ are affected by emission due to the source itself and will not be further discussed here. The two additional sets of $\mathrm{HF}$ absorption components in the ranges $V_{\mathrm{LSR}}=$ $30-50$ and $50-75 \mathrm{~km} \mathrm{~s}^{-1}$ are unrelated to the source and have been detected through absorption from other species such as $\mathrm{HCO}^{+}, \mathrm{HCN}, \mathrm{HNC}$ (Godard et al. 2010), or atomic hydrogen (Fish et al. 2003) and atomic oxygen (Vastel et al. 2000). As for G10.6-0.4 (W31C) observed previously by Neufeld et al. (2010), the profiles of HF and para-water are remarkably similar with the exception of two components at LSR velocities of $\sim 68$ and $71 \mathrm{~km} \mathrm{~s}^{-1}$ that are clearly much stronger in HF than in water.

The distributions of foreground material toward W51 also exhibit a one-to-one correspondence between the HF and parawater absorptions with the exception of one component at LSR velocity of $24 \mathrm{~km} \mathrm{~s}^{-1}$ that is clearly detected in HF, but is absent in the para-water spectrum. This component at $24 \mathrm{~km} \mathrm{~s}^{-1}$ has been detected in the absorption spectra of atomic hydrogen (Koo 1997) and the HIFI spectra of $\mathrm{CH}^{+}$(Falgarone et al. 2010), but to our knowledge - has not been observed in studies of molecular absorption prior to the launch of Herschel.

The lower panels of Figs. 1 and 2 compare the HF antenna temperature $T_{\mathrm{A}}(\mathrm{K})$ with the para-water antenna temperature. Here, each point represents one velocity bin in those LSR velocity ranges exhibiting only moderate HF and para-water optical depths. The dashed lines represent the continuum temperature $T_{\mathrm{A}}$ (cont) $(\mathrm{K})$ and $0.5 T_{\mathrm{A}}$ (cont) for $\mathrm{HF}$ (vertical dashed lines) and para-water (horizontal dashed lines). The solid black lines in the lower panels of Figs. 1 and 2 represent the expected location of the HF and para-water antenna temperatures in each velocity bin for a given optical depth ratio of HF over para-water. From top to bottom, we display HF to para-water ratios of 5, 3, 2, 1, 0.5, 0.33 and 0.2. For G10.6-0.4 (Neufeld et al. 2010), we find that the optical depth of HF is between 2 and 5 times larger than that of para-water toward both sight lines. Note again the presence of the $24 \mathrm{~km} \mathrm{~s}^{-1}$ component detected in HF and clearly absent in para-water (blue diamonds).

\section{Discussion}

Toward W51, all para-water components in the LSR velocity range 0 to $30 \mathrm{~km} \mathrm{~s}^{-1}$ are optically thin, while those of HF are either optically thin (the 12 and $24 \mathrm{~km} \mathrm{~s}^{-1}$ components) or moderately thick (the 6 and the $45 \mathrm{~km} \mathrm{~s}^{-1}$ complexes), hence allowing us to directly measure the column density of these species for each set of clouds. This stands in contrast to G10.6-0.4, where the large HF $J=1-0$ optical depth allowed Neufeld et al. (2010) to obtain only a lower limit on the HF abundance. Note that the HF and para-water lines are optically thick in the velocity range 50 to $75 \mathrm{~km} \mathrm{~s}^{-1}$, thus only leading to lower limits on their column densities.

We used a set of multiple Gaussian components to simultaneously fit the HF and para-water profiles in the $0-30 \mathrm{~km} \mathrm{~s}^{-1}$ range. Due to the similarity between the $\mathrm{HF}$ and $\mathrm{HCO}^{+}$profiles in this velocity range, we used the $\mathrm{HCO}^{+}$sight line cloud decomposition of Godard et al. (2010) as an initial guess in our fits. Under the assumption that both HF and para-water co-exist along the W51 sight line, we fixed the cloud velocity and FWHM of the para-water components to equal those of HF.

Figure 3 displays the optical depth spectra of HF (black line) and para-water (green line) in the -5 to $30 \mathrm{~km} \mathrm{~s}^{-1}$ velocity range 

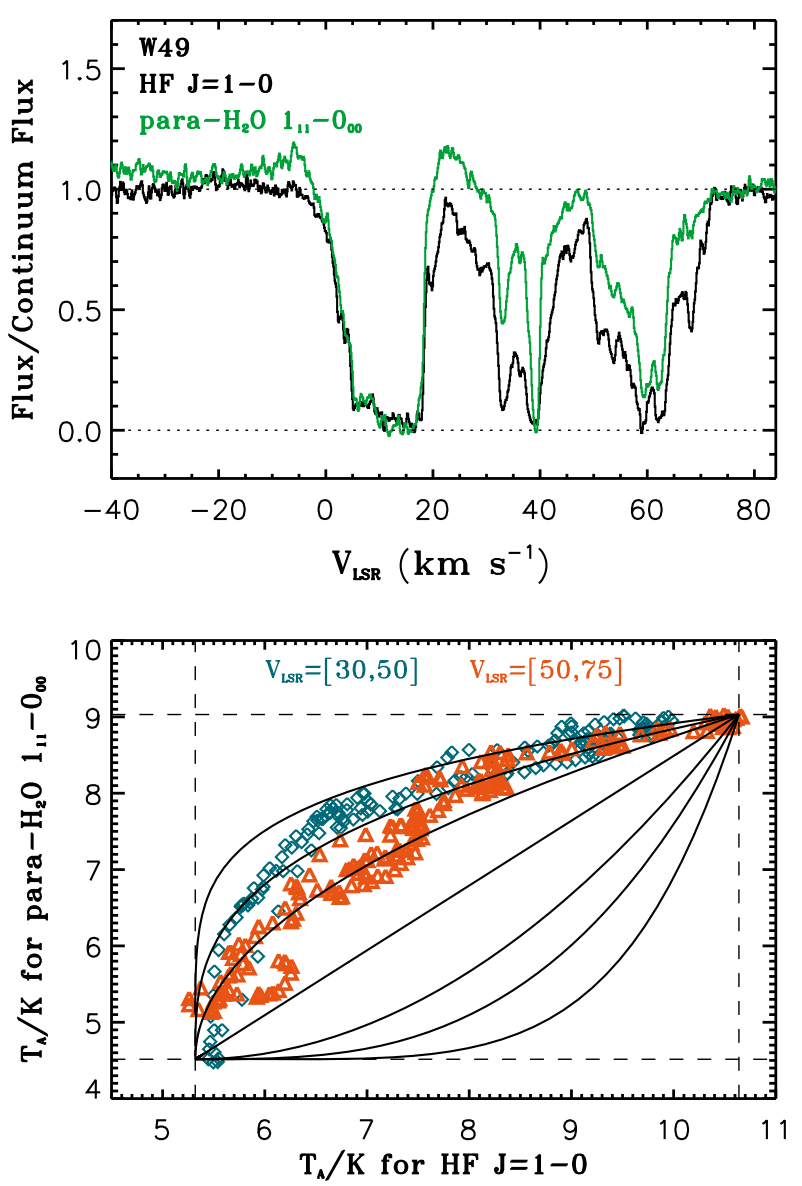

Fig. 1. Top: normalized spectra of $\mathrm{HF}$ and para- $\mathrm{H}_{2} \mathrm{O}$ over $V_{\mathrm{LSR}}=$ $[-40,85] \mathrm{km} \mathrm{s}^{-1}$ (see Sect. 2 for details). Bottom: HF $J=1-0$ antenna temperature versus para- $\mathrm{H}_{2} \mathrm{O} 1_{11}-0_{00}$ antenna temperature over the velocity ranges indicated at the top of the figure toward the W49N sight line. The solid black lines represent the expected loci for given optical depth ratios of $\mathrm{HF}$ to para-water of 5, 3, 2, 1, 0.5, 0.33, 0.2 from top to bottom.

toward W51, with the best fit to each spectrum superimposed as a red line. The quality of both fits clearly demonstrates that our assumption regarding the similarity in distribution for $\mathrm{HF}$ and para-water is justified for the cloud complex at $6 \mathrm{~km} \mathrm{~s}^{-1}$ and the $12 \mathrm{~km} \mathrm{~s}^{-1}$ component. This figure further shows that the distribution of $\mathrm{HCO}^{+}$does differ slightly from that of $\mathrm{HF}$ for the $6 \mathrm{~km} \mathrm{~s}^{-1}$ cloud complex and that the $24 \mathrm{~km} \mathrm{~s}^{-1}$ component is traced by $\mathrm{HF}$ alone. The $\mathrm{HCO}^{+}$counterpart of the $45 \mathrm{~km} \mathrm{~s}^{-1}$ complex is blended with strong emission, preventing Godard et al. (2010) from deriving the cloud structure for this absorption complex. In addition the blending between the various features seen in our data is too severe to lead to a unique fitting solution. We therefore integrated the $\mathrm{HF}$ and water absorption profiles over the entire $42-47 \mathrm{~km} \mathrm{~s}^{-1}$ range (see Gerin et al. 2010).

Toward W49N, the HF and para-water cloud complexes in the $30-50$ and $50-65 \mathrm{~km} \mathrm{~s}^{-1}$ ranges are all optically thicker than those toward W51. Additionally, the various cloud components detected in $\mathrm{HCO}^{+}$by Godard et al. (2010) are more severely blended than toward W51, preventing us from obtaining a unique fit to the cloud distributions for W49N. Consequently, we integrated the HF and para-water optical depths over the entire
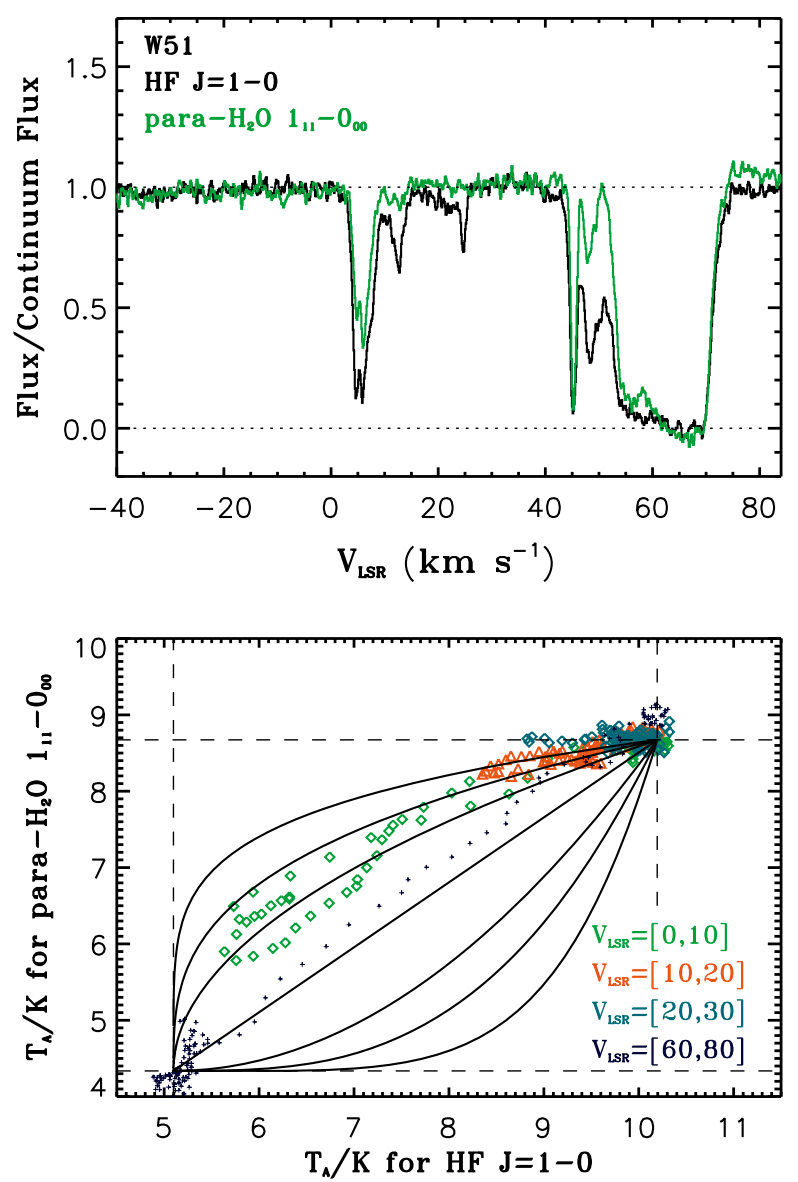

Fig. 2. Top: normalized spectra of $\mathrm{HF}$ and para- $\mathrm{H}_{2} \mathrm{O}$ over $V_{\mathrm{LSR}}=[-40$, 85] $\mathrm{km} \mathrm{s}^{-1}$. Bottom: $\mathrm{HF} J=1-0$ antenna temperature versus para- $\mathrm{H}_{2} \mathrm{O}$ $1_{11}-0_{00}$ antenna temperature over the velocity ranges indicated in the lower right corner toward the W51 sight line. Note the absence of parawater absorption compared to HF for $V_{\mathrm{LSR}}=[20,30] \mathrm{km} \mathrm{s}^{-1}$ (blue diamonds). The solid black lines represent the expected loci for given optical depth ratios of HF to para-water of 5, 3, 2, 1, 0.5, 0.33, 0.2 from top to bottom.

velocity ranges with the exception of the two absorbing clouds at LSR velocities of 68 and $71 \mathrm{~km} \mathrm{~s}^{-1}$. These two components are optically thin in both HF and para-water and were fitted with Gaussians using the same method as for W51.

Following Neufeld et al. (2010), we derived the HF and parawater column densities for each LSR velocity range by dividing the velocity-integrated optical depths of $\mathrm{HF}$ and para-water by $4.16 \times 10^{-13} \mathrm{~cm}^{2} / \mathrm{km} \mathrm{s}^{-1}$ and $4.30 \times 10^{-13} \mathrm{~cm}^{2} / \mathrm{km} \mathrm{s}^{-1}$, respectively, to obtain the results given in Table 1 . Here, we assume that the absorbing material completely covers the continuum emission region, and that each molecule is primarily in its ground state. The latter assumption is justified because the gas density is much lower than the critical density at which the collisional deexcitation and spontaneous decay rates would be equal (Neufeld et al. 2010). For the same velocity ranges, we have presented estimates for the $\mathrm{H}_{2}$ and atomic $\mathrm{H}$ column densities. In diffuse molecular clouds (also known as "translucent clouds" in the classification proposed by Snow \& McCall 2006), direct measurements of the $\mathrm{H}_{2}$ and $\mathrm{CH}$ column density via far- and near-UV absorption spectroscopy showed that molecular hydrogen and $\mathrm{CH}$ trace each other linearly, with 


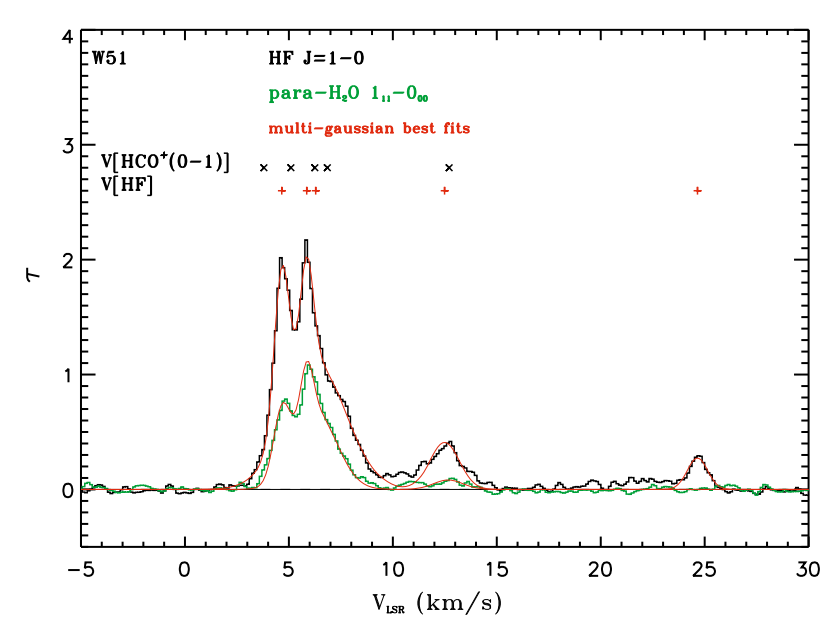

Fig. 3. Optical depth spectra of HF $J=1-0$ (black line) and para-water $1_{11}-0_{00}$ (green line) toward W51. The (x) mark the positions of the $\mathrm{HCO}^{+}$components (see Godard et al. 2010). The (+) mark the positions of HF components resulting from our multi-component Gaussian fit to the HF data. When fitting the para-water spectrum, the component positions and $F W H M$ were held fixed at the values derived from the $\mathrm{HF}$ analysis.

$N(\mathrm{CH})=3.5 \times 10^{-8} N\left(\mathrm{H}_{2}\right)$ (Sheffer et al. 2008, and references therein). Hence, $\mathrm{CH}$ is often used as proxy for $\mathrm{H}_{2}$ when the latter cannot be measured directly. Gerin et al. (2010) detected absorption of $\mathrm{CH}(v=536.761 \mathrm{GHz})$ toward both W49N and W51. The distribution of the $\mathrm{CH}$ absorbing clouds matches that of $\mathrm{HF}$ toward both sight lines. We therefore used the $\mathrm{CH}$ column densities derived by Gerin et al. (2010) and the Sheffer et al. (2008) relationship to infer the $\mathrm{H}_{2}$ column densities toward $\mathrm{W} 49 \mathrm{~N}$ and W51. The uncertainty in our determination of the $\mathrm{H}_{2}$ column density is dominated by the scatter in the $\mathrm{CH}-\mathrm{H}_{2}$ relationship, estimated by Sheffer et al. as 0.21 dex, corresponding to a factor of 1.6. The atomic hydrogen column densities for W51 were obtained from the $21 \mathrm{~cm}$ absorption spectra obtained by Koo (1997) for an assumed $21 \mathrm{~cm}$ spin temperature of $100 \mathrm{~K}$, while those for W49N were obtained by Godard et al. (2010), based upon $21 \mathrm{~cm}$ observations presented by Fish et al. (2003).

In their analysis of the chemistry of fluorine-containing molecules in the interstellar medium, Neufeld \& Wolfire (2009) predicted (e.g. their Fig. 7) that over a wide range of conditions the $\mathrm{HF}$ and $\mathrm{H}_{2}$ column densities would track each other exactly, with the ratio $N(\mathrm{HF}) / 2 N\left(\mathrm{H}_{2}\right)$ equal to the gas-phase elemental abundance of $\mathrm{F}$ relative to $\mathrm{H}$ nuclei. Given the average gas-phase abundance of interstellar fluorine in diffuse atomic gas of $N_{\mathrm{F}} / N_{\mathrm{H}}=1.8 \times 10^{-8}$ (Snow et al. 2007), this result implies a $N(\mathrm{HF}) / N\left(\mathrm{H}_{2}\right)$ ratio of $3.6 \times 10^{-8}$. Given the uncertainty in the $N(\mathrm{CH})-N\left(\mathrm{H}_{2}\right)$ relationship used to infer the molecular hydrogen column densities and the variations in the gas-phase fluorine abundance, the results shown in Table 1 are consistent with that prediction. The lack of correlation between $N(\mathrm{HF})$ and $N(\mathrm{H})$ for the cloud components presented in this work also suggests that $\mathrm{HF}$ is absent in purely atomic material, but has the potential to serve as an excellent tracer of $\mathrm{H}_{2}$. Hydrogen fluoride provides a sensitive probe of clouds of small $\mathrm{H}_{2}$ column density. Indeed, the observations of HF reported here reveal a low column density molecular cloud along the W51 sight line, at an LSR velocity of $\sim 24 \mathrm{~km} \mathrm{~s}^{-1}$, which had not been identified in molecular absorption line studies prior to the launch of Herschel.
Table 1. Summary of derived column densities and abundances.

\begin{tabular}{cccccc}
\hline \hline $\begin{array}{c}V_{\text {LSR }} \\
\left(\mathrm{km} \mathrm{s}^{-1}\right)\end{array}$ & $\begin{array}{c}N(\mathrm{HF}) \\
10^{12} \mathrm{~cm}^{-2}\end{array}$ & $\begin{array}{c}N\left(\mathrm{H}_{2} \mathrm{O}\right) \\
10^{12} \mathrm{~cm}^{-2}\end{array}$ & $\begin{array}{c}N(\mathrm{H})^{a} \\
10^{20} \mathrm{~cm}^{-2}\end{array}$ & $\begin{array}{c}N\left(\mathrm{H}_{2}\right)^{b} \\
10^{20} \mathrm{~cm}^{-2}\end{array}$ & $\mathrm{HF} / \mathrm{H}_{2}$ \\
\hline \multicolumn{7}{l}{ Results for W51 } \\
$0-10$ & $14.5 \pm 1.1$ & $6.2 \pm 0.5$ & 13.9 & 10.5 & $1.4 \times 10^{-8}$ \\
$10-20$ & $1.8 \pm 0.3$ & $0.4 \pm 0.1$ & 6.4 & 1.4 & $1.3 \times 10^{-8}$ \\
$20-30$ & $0.8 \pm 0.1$ & $<0.2$ & 11.1 & $<0.7$ & $>1.2 \times 10^{-8}$ \\
$42-47$ & $8.0 \pm 1.0$ & $5.4 \pm 0.7$ & $\ldots$ & 5.0 & $1.6 \times 10^{-8}$ \\
$50-75$ & $>130$ & $>113$ & 22.1 & $\ldots$ & $\ldots$ \\
\multicolumn{7}{l}{ Results for W49N } & & & & \\
$30-50$ & $55 \pm 10$ & $22 \pm 8$ & 69.5 & 37 & $1.5 \times 10^{-8}$ \\
$50-78$ & $69 \pm 10$ & $34 \pm 9$ & 72.3 & 66 & $1.1 \times 10^{-8}$ \\
$67-71$ & $5.6 \pm 1.0$ & $1.5 \pm 0.3$ & $\ldots$ & 4.4 & $1.3 \times 10^{-8}$ \\
\hline
\end{tabular}

Notes. ${ }^{(a)}$ from Koo 1997 (W51) and Godard et al. 2010 (W49N), for an assumed $21 \mathrm{~cm}$ spin temperature of $100 \mathrm{~K}$.

(b) Derived from $\mathrm{CH}$ observations (Gerin et al. 2010), assuming $N(\mathrm{CH})=3.5 \times 10^{-8} N\left(\mathrm{H}_{2}\right)$ (Sheffer et al. 2008). The relationship shows a scatter of 0.21 dex, corresponding to a factor of 1.6.

Acknowledgements. HIFI has been designed and built by a consortium of institutes and university departments from across Europe, Canada and the United States under the leadership of SRON Netherlands Institute for Space Research, Groningen, The Netherlands and with major contributions from Germany, France and the US. Consortium members are: Canada: CSA, U. Waterloo; France: CESR, LAB, LERMA, IRAM; Germany: KOSMA, MPIfR, MPS; Ireland, NUI Maynooth; Italy: ASI, IFSI-INAF, Osservatorio Astrofisico di Arcetri- INAF; Netherlands: SRON, TUD; Poland: CAMK, CBK; Spain: Observatorio Astronómico Nacional (IGN), Centro de Astrobiologá (CSIC-INTA). Sweden: Chalmers University of Technology - MC2, RSS \& GARD; Onsala Space Observatory; Swedish National Space Board, Stockholm University - Stockholm Observatory; Switzerland: ETH Zurich, FHNW; USA: Caltech, JPL, NHSC. M.S. acknowledges support from grant N 203393334 from Polish MNiSW.

This research was performed in part through a JPL contract funded by the National Aeronautics and Space Administration.

\section{References}

de Graauw, Th., Helmich, F., Phillips, T., et al. 2010, A\&A, 518, L6 Falgarone, E., Godard, B., Cernicharo, J., et al. 2010, A\&A, 521, L15 Fish, V. L., Reid, M. J., Wilner, D. J., \& Churchwell, E. 2003, ApJ, 587, 701 Godard, B., Falgarone, E., Gerin, M., Hily-Brant, P., \& De Luca, M. 2010, A\&A, 520, A20

Gerin, M., De Luca, M., Goicoechea, J. R., et al. 2010, A\&A, 521, L16 Gwinn, C. R., Moran, J. M., \& Reid, M. J. 1992, ApJ, 393, 149 Kirk, J. M., Polehampton, E., Anderson, L. D., et al. 2010, A\&A, 518, L82 Koo, B.-C. 1997, ApJS, 108, 489

Neufeld, D. A., \& Wolfire, M. 2009, ApJ, 706, 1594

Neufeld, D. A., Kaufman, M. J., Goldsmith, P. F., Hollenbach, D. J., \& Plume, R. 2002, ApJ, 580, 278

Neufeld, D. A., Wolfire, M., \& Schilke, P. 2005, ApJ, 628, 260

Neufeld, D. A., Sonnentrucker, P., Phillips, T. G., et al. 2010, A\&A, 518, L108 Nolt, I. J., Radostitz, J. V., Dilonardo, G., et al. 1987, J. Molecular Spectroscopy, 125,274

Ott, S. 2010, in Astronomical Data Analysis Software and Systems XIX, ed. Y. Mizumoto, K.-I. Morita, \& Ohishi, ASP Conf. Ser., in press Phillips, T. G., Bergin, E. A., Lis, D. C., et al. 2010, A\&A, 518, L109 Pilbratt, G., Riedinger, J. R., Passvogel, T., et al. 2010, A\&A, 518, L1 Plume, R., Kaufman, M. J., Neufeld, D. A., et al. 2004, ApJ, 605, 247 Sato, M., Reid, M. J., Brunthaler, A., \& Menten, K. M. 2010, ApJ, 720, 1055 Sheffer, Y., Rogers, M., Federman, S. R., et al. 2008, ApJ, 687, 1075 Snow, T. P., \& McCall, B. J. 2006, ARA\&A, 44, 367

Snow, T. P., Destree, J. D., \& Jensen, A. G. 2007, ApJ, 655, 285 Vastel, C., Caux, E., Ceccarelli, C., et al. 2000, A\&A, 357, 994 
1 The Johns Hopkins University, Baltimore, MD 21218, USA e-mail: sonnentr@pha.jhu.edu

2 California Institute of Technology, Pasadena, CA 91125, USA

${ }^{3}$ LERMA, CNRS, Observatoire de Paris and ENS, France

4 Centro de Astrobiologìa, CSIC-INTA, 28850 Madrid, Spain

5 Chalmers University of Technology, Göteborg, Sweden

${ }^{6}$ Institut d'Astrophysique Spatiale (IAS), Orsay, France

7 Université Toulouse; UPS; CESR; and CNRS; UMR5187, 9 avenue du colonel Roche, 31028 Toulouse cedex 4, France

8 Nicolaus Copernicus University, Torún, Poland

9 Gemini telescope, Hilo, Hawaii, USA

10 I. Physikalisches Institut, University of Cologne, Germany
11 JPL, California Institute of Technology, Pasadena, USA

12 LAM, OAMP, Université Aix-Marseille \& CNRS, Marseille, France

13 Depts. of Physics, Astronomy \& Chemistry, Ohio State Univ., USA

14 Laboratoire d'Astrophysique de Grenoble, France

15 Institute of Physical Chemistry, PAS, Warsaw, Poland

16 MPI für Radioastronomie, Bonn, Germany

17 Tata Institute of Fundamental Research, Homi Bhabha Road, Mumbai 400005, India

18 Dept. of Physics \& Astronomy, University of Calgary, Canada

19 Nicolaus Copernicus Astronomical Center, Torún, Poland

${ }^{20}$ European Space Astronomy Centre, ESA, Madrid, Spain

${ }^{21}$ Infrared Processing and Analysis Center, California Institute of Technology, MS 100-22, Pasadena, CA 91125, USA 